\title{
ON PARTIALLY CONSERVATIVE SENTENCES AND INTERPRETABILITY
}

\begin{abstract}
PER LINDSTROM
ABSTRACT. A sentence $\varphi$ is $\Gamma$-conservative over $T$ if $T+\varphi \vdash \psi$ implies $T \vdash \psi$ for every $\psi \in \Gamma$. In $\S 1$ this concept for $\Gamma=\Sigma_{n+1}^{0}$ and $\Pi_{n+1}^{0}$ is investigated. In $\S 2$ results from $\S 1$ are applied to interpretability in theories containing arithmetic.
\end{abstract}

0. Introduction. Let $\Gamma$ be a set of sentences. A sentence $\varphi$ is $\Gamma$-conservative over a theory $T, \varphi \in \operatorname{Cons}(\Gamma, T)$, if $T+\varphi \vdash \psi$ implies $T \vdash \psi$ for every $\psi \in \Gamma$. This concept was introduced by Guaspari [2] and the basic existence theorems were established by him and Solovay (cf. [2]). The first example of a result of this type is, however, due to Kreisel [7] who observed that if $\operatorname{Con}_{P}$ is a "natural" formalization of " $\mathrm{P}$ (eano arithmetic) is consistent", then $\neg_{\neg} \operatorname{Con}_{P} \in \operatorname{Cons}\left(\Pi_{1}^{0}, P\right)$. Related results have also been obtained by Hájek [4], Jensen and Ehrenfeucht [6], and Kreisel and Lévy [8]. Subsequently the results of Guaspari and Solovay were somewhat improved and their proofs were simplified by Hájek [4], Smoryński [14, 15], and the author. The general method of proof applied in the papers mentioned as well as in the present paper is, however, the same, namely a combination of partial truth definitions and self-reference.

In $\$ 1$ we present two groups of results. Those belonging to the first group (Theorems 1-5 and Corollary 1) concern the existence of partially conservative sentences and partially conservative extensions of theories satisfying various additional conditions. In the results of the second group (Theorems 6 and 7 and Corollaries 2 and 3 ) it is shown that a number of sets occurring naturally in the study of partial conservativity are complete at certain levels of the arithmetical hierarchy. In $\$ 2$ results from $\$ 1$ are applied to (relative) interpretability using the fact that if $S$ and $T$ are reflexive r.e. extensions of $P$, then $S$ is interpretable in $T$ iff every $\Pi_{1}^{0}$ sentence provable in $S$ is provable in $T$. In particular we answer two questions raised by Orey [12] and Švejdar [17]. For further applications of partially conservative sentences to interpretability see $[10,11]$.

1. Partially conservative sentences. In the following $\Gamma$ is either $\Sigma_{n+1}^{0}$ or $\Pi_{n+1}^{0} . \breve{\Gamma}$ is the dual of $\Gamma$. $A$ and $B$ are consistent primitive recursive extensions of $P$. (By Craig's theorem (cf. [1]), every r.e. set of $(\Gamma)$ sentences is deductively equivalent to a primitive recursive set of $(\Gamma)$ sentences.) $\operatorname{Th}(T)=\{\varphi: T \vdash \varphi\}$. We write $T \vdash X$ or

Received by the editors October 14, 1982 and, in revised form, May 16, 1983.

1980 Mathematics Subject Classification. Primary 03F25, 03F30. 
$X \dashv T$ to mean that $X \subseteq \operatorname{Th}(T)$. $S$ is an $X$-subtheory of $T, S \dashv_{X} T$, if $\operatorname{Th}(S) \cap X \subseteq$ $\operatorname{Th}(T) . \varphi^{i}$ is $\varphi$ if $i=0$ and $\neg \varphi$ if $i=1$. The diagonal lemma (self-reference) will be used repeatedly without further comment. For notation and terminology not explained here see [1].

As is well known, to each $\Gamma$ there is a $\Gamma$ formula $\Gamma$-true $(x)$ s.t. for every $\varphi \in \Gamma$,

$$
P \vdash \varphi \leftrightarrow \Gamma \text {-true }(\bar{\varphi}) \text {. }
$$

Let $\alpha(x)$ be a PR binumeration of $A$ and let $\{\Gamma\}(x, y)$ be the formula

$$
\forall u v \leqslant y\left(u \text { is } \Gamma \wedge \operatorname{Prf}_{\alpha(z) \vee z=x}(u, v) \rightarrow \Gamma \text {-true }(u)\right)
$$

(cf. $[2,4,6,8,14])$. The following lemma is then easily verified.

LEMMA $1 .\{\Gamma\}(x, y)$ is a $\Gamma$ formula s.t.

(i) $P \vdash\{\Gamma\rangle(x, y) \wedge y^{\prime} \leqslant y \rightarrow\{\Gamma\}\left(x, y^{\prime}\right)$,

(ii) $A+\varphi \vdash\{\Gamma\}(\bar{\varphi}, \bar{m})$, all $\varphi$ and $m$,

(iii) if $\psi$ is $\Gamma$ and $A+\varphi \vdash \psi$, then there is a q s.t. $P+\{\Gamma\rangle(\bar{\varphi}, \bar{q}) \vdash \psi$.

The next two lemmas serve to unify a number of proofs in what follows.

Lemma 2. Suppose $\chi(x, y)$ is $\Gamma$. Then there is a $\Gamma$ formula $\xi(x)$ s.t.

(i) $A+\xi(\bar{k}) \vdash \chi(\bar{k}, \bar{m})$,

(ii) if $B \dashv A$, then $B+\xi(\bar{k}) \dashv \check{\Gamma} B \cup\{\chi(\bar{k}, \bar{q}): q \in \omega\}$.

Proof. Case 1. $\Gamma=\Pi_{n+1}^{0}$. Let $\xi(x)$ be s.t.

$$
P \vdash \xi(\bar{k}) \leftrightarrow \forall y\left(\left\{\Sigma_{n+1}^{0}\right\}(\overline{\xi(\bar{k})}, y) \rightarrow \chi(\bar{k}, y)\right) .
$$

Then (i) follows at once from Lemma 1(ii). To prove (ii), suppose $\psi$ is $\Sigma_{n+1}^{0}, B \dashv A$, and $B+\xi(\bar{k}) \vdash \psi$. By Lemma 1(iii), there is a $q$ s.t.

$$
P+\left\{\Sigma_{n+1}^{0}\right\}(\overline{\xi(\bar{k})}, \bar{q}) \vdash \psi \text {. }
$$

Hence, by Lemma 1(i),

$$
P+\forall y \leqslant \bar{q} \chi(\bar{k}, y)+\neg \psi \vdash \xi(\bar{k}) .
$$

But then, since $B+\xi(\bar{k}) \vdash \psi$, it follows that $B+\forall y \leqslant \bar{q} \chi(\bar{k}, y) \vdash \psi$.

Case 2. $\Gamma=\Sigma_{n+1}^{0}$. Let $\xi(x)$ be s.t.

$$
P \vdash \xi(\bar{k}) \leftrightarrow \exists y\left(\neg\left\{\Pi_{n+1}^{0}\right\}(\overline{\xi(\bar{k})}, y) \wedge \forall z \leqslant y \chi(\bar{k}, z)\right) .
$$

The proof that $\xi(x)$ is as claimed is almost the same as in Case 1.

Lemma 3. Suppose $\chi_{0}(x, y)$ is $\breve{\Gamma}$ and $\chi_{1}(x, y)$ is $\Gamma$. Then there is $a \Gamma$ formula $\xi(x)$ s.t. for $i=0,1$

(i) $A+\xi^{i}(\bar{k}) \vdash \forall y \leqslant \bar{m} \chi_{i}(\bar{k}, y) \rightarrow \chi_{1-i}(\bar{k}, \bar{m})$,

(ii) if $\psi$ is $\breve{\Gamma}$ and $A+\xi^{i}(\bar{k}) \vdash \psi^{i}$, then $A \cup\left\{\chi_{1-i}(\bar{k}, \bar{q}): q \in \omega\right\} \vdash \psi^{i}$.

Proof. We need only prove this for $\Gamma=\Sigma_{n+1}^{0}$. Let $\xi(x)$ be s.t.

$$
\begin{aligned}
& P \vdash \xi(\bar{k}) \leftrightarrow \exists y\left(\left(\neg\left\{\Pi_{n+1}^{0}\right\}(\overline{\xi(\bar{k})}, y) \vee \neg \chi_{0}(\bar{k}, y)\right) \wedge\right. \\
& \left.\forall z<y\left(\left\{\Sigma_{n+1}^{0}\right\}(\overline{\neg \xi(\bar{k})}, z) \wedge \chi_{1}(\bar{k}, z)\right)\right) .
\end{aligned}
$$

The proof of Lemma 3 is now very much the same as the proof of Lemma 2. 
Let us say that $\varphi$ is hereditarily $\Gamma$-conservative over $A, \varphi \in \operatorname{HCons}(\Gamma, A)$, if $\varphi \in \operatorname{Cons}(\Gamma, B)$ for every $B \dashv A$. The following result is due to Guaspari [2] (cf. also $[4,14,15])$.

THEOREM 1. Let $X$ be any r.e. set. There is then a $\Gamma$ formula $\xi(x)$ s.t.

(i) if $k \in X$, then $A \vdash \neg \xi(\bar{k})$,

(ii) if $k \notin X$, then $\xi(\bar{k}) \in \operatorname{HCons}(\breve{\Gamma}, A)$.

Proof. Let $\rho(x, y)$ be a PR binumeration of a relation $R(k, m)$ s.t. $X=\{k: \exists m$ $R(k, m)\}$ and let $\xi(x)$ be as in Lemma 2 with $\chi(x, y)=\neg \rho(x, y)$. Then (i) follows from Lemma 2(i) and (ii) follows from Lemma 2(ii).

A set $X$ of sentences is mono-consistent with $T$ if $T+\varphi$ is consistent for every $\varphi \in X$ (cf. [9]).

CoRollary 1. If $X$ is r.e. and mono-consistent with $A$, then $\Gamma \cap \operatorname{HCons}(\check{\Gamma}, A)-X$ $\neq \varnothing$.

Proof. Let $\xi(x)$ be as in Theorem 1 and let $\varphi$ be s.t. $P \vdash \varphi \leftrightarrow \xi(\bar{\varphi})$. If $\varphi \in X$, then $A \vdash \neg \xi(\bar{\varphi})$, whence $A \vdash \neg \varphi$. But this is impossible and so $\varphi \notin X$. But then, by Theorem 1(ii), $\varphi$ is as desired.

Let $N X=\{\varphi: \neg \varphi \in X\}$ and $\operatorname{DCons}(\Gamma, A)=\operatorname{Cons}(\Gamma, A) \cap \operatorname{NCons}(\check{\Gamma}, A)$. Solovay proved that $\Gamma \cap \operatorname{DCons}(\check{\Gamma}, A) \neq \varnothing$ (cf. [2]). This can be improved as follows (cf. $[14,15])$.

THEOREM 2. Suppose $X$ is r.e. and mono-consistent with $A$. Then $\Gamma \cap \operatorname{DCons}(\check{\Gamma}, A)$ $-(X \cup N X) \neq \varnothing$.

Proof. Let $\rho_{i}(x, y)$ be PR binumerations of relations $R_{i}(k, m)$ s.t. $X=\{k: \exists m$ $\left.R_{0}(k, m)\right\}$ and $N X=\left\{k: \exists m R_{1}(k, m)\right\}$. Let $\xi(x)$ be as in Lemma 3 with $\chi_{i}(x, y)=$ $\neg \rho_{i}(x, y)$ and let $\theta$ be s.t. $P \vdash \theta \leftrightarrow \xi(\bar{\theta})$. Suppose $\theta \in X \cup N X$. Let $n$ be the least number s.t. $R_{0}(\theta, n)$ or $R_{1}(\theta, n)$. Suppose $R_{i}(\theta, n)$. Then not $R_{1-i}(\theta, m)$ for $m \leqslant n$. (We may assume that $R_{0}(k, m)$ implies not $R_{1}(k, m)$.) Hence, by Lemma 3(i), $A \vdash \neg \xi^{i}(\bar{\theta})$, whence $A \vdash \neg \theta^{i}$, which is impossible since $\theta^{i} \in X$. Thus $\theta \notin X \cup N X$. But then, by Lemma 3(ii), $\theta \in \operatorname{DCons}(\check{\Gamma}, A)$.

The result of Solovay mentioned above together with Corollary 1 with $X=\mathrm{Th}(A)$ led to the question if $Y=\cap\{\operatorname{DCons}(\Gamma, B): B \dashv A\} \neq \varnothing$ (cf. [2, 14]). It is easily seen, however, that this is not true in general. Let $\varphi$ be a $\Gamma$ sentence and $\psi$ a $\breve{\Gamma}$ sentence s.t. $P \nvdash \varphi^{i} \vee \psi^{i}, i=0,1$, and let $A=P+\varphi \wedge \psi$. Suppose $\theta \in Y$. Then, since $P+\theta \rightarrow \varphi+\theta \vdash \varphi$, we get $P+\theta \rightarrow \varphi \vdash \varphi$ and so $P+\neg \theta \vdash \varphi$. Similarly $P+\theta \vdash \psi$. But then $P \vdash \varphi \vee \psi$, contrary to hypothesis.

Our next result will be applied in $\$ 2$ to answer a question of Orey [12].

THEOREM 3. There are sentences $\varphi_{i}$ s.t. $\varphi_{i}, \neg\left(\varphi_{0} \wedge \varphi_{1}\right) \in \operatorname{Cons}(\Gamma, A)-$ $\operatorname{NCons}\left(\Pi_{1}^{0}, A\right), i=0,1$.

We write $A \stackrel{p}{\dashv}{ }_{X} B$ (" $p$ " for "proper") to mean that $A \dashv{ }_{X} B{ }^{\prime}{ }_{X} A$.

Lemma 4. Suppose $A \dashv B H_{\Pi_{1}^{0}} A$. Then there is a sentence $\chi$ s.t. $A \stackrel{p}{\dashv} \Pi_{1}^{0} A+$ $\chi^{i} \dashv_{\Gamma} B, i=0,1$. 
Proof. Let $\theta$ be a $\Pi_{1}^{0}$ sentence s.t. $B \vdash \theta$ and $A \nvdash \theta$ and let $\theta_{i}$ be s.t.

$$
P \vdash \theta_{i} \leftrightarrow \forall z\left(\operatorname{Prf}_{\alpha}\left(\overline{\theta \vee \theta_{i}}, z\right) \rightarrow \exists u \leqslant_{i} z \operatorname{Prf}_{\alpha}\left(\overline{\theta \vee \theta_{1-i}}, u\right)\right),
$$

where $\leqslant_{0}$ is $\leqslant$ and $\leqslant_{1}$ is $<$ (cf. [6]). Then, by a standard argument,

(1) $P \vdash \theta_{0} \vee \theta_{1}$,

(2) $A \nLeftarrow \theta \vee \theta_{i}$.

Suppose $\Gamma \cup \check{\Gamma} \subseteq \Gamma^{*}$. By Lemma 3, there is a sentence $\chi$ s.t.

(3) $A+\chi^{i} \vdash\left(\theta \vee \theta_{i}\right) \rightarrow\left(\theta \vee \theta_{1-i}\right)$,

(4) if $\psi$ is $\Gamma^{*}$ and $A+\chi^{i} \vdash \psi^{i}$, then $A+\left(\theta \vee \theta_{1-i}\right) \vdash \psi^{i}$.

By (1), (2), (3), $A \dashv \Pi_{1}^{0} A+\chi^{i}$. Finally, by (4), $A+\chi^{i} \dashv \Gamma_{\Gamma} B$.

Proof of Theorem 3. Let $\eta$ and $\theta$ be s.t. $\eta^{i} \notin \operatorname{Cons}\left(\Pi_{1}^{0}, A\right)$ and $\theta^{i} \in \operatorname{Cons}(\Gamma, A)$, $i=0,1$ (cf. [17] and Theorem 2). Let $\psi=\eta \wedge \theta$. Then

(1) $\psi \in \operatorname{NCons}(\Gamma, A)-\operatorname{Cons}\left(\Pi_{1}^{0}, A\right)$,

(2) $\psi \vee \neg \eta \in \underset{p}{\operatorname{Cons}}(\Gamma, A)$.

Moreover $A \dashv \Pi_{1}^{0} A+\neg \eta$. So, by Lemma 4, there is a sentence $\chi$ s.t.

(3) $A \stackrel{p}{\dashv} \Pi_{1}^{0} A+\chi^{i} \dashv{ }_{\Gamma} A+\neg \eta$.

Finally let $\varphi_{i}$ be $\psi \vee \chi^{i}$. Then, by (2) and (3), $\varphi_{i} \in \operatorname{Cons}(\Gamma, A)-\operatorname{NCons}\left(\Pi_{1}^{0}, A\right)$. Moreover $\vdash\left(\varphi_{0} \wedge \varphi_{1}\right) \leftrightarrow \psi$. So, by (1), $\varphi_{0}$ and $\varphi_{1}$ are as desired.

The next two results are refinements of the following simple and certainly well-known observation. Suppose $X$ is r.e., bounded, i.e. $X \subseteq \Gamma$ for some $\Gamma$, and $A \cup X$ is consistent. Then there is a sentence $\theta$ s.t. $A \cup X \dashv A+\theta$ and $A+\theta$ is consistent. This can be improved as follows. Let us say that $S$ is a $\Gamma$-conservative extension of $T$ if $T \dashv S \dashv{ }_{\Gamma} T$.

THEOREM 4. Let $X$ be an r.e. set of $\Gamma$ sentences. Then there is a $\Gamma$ sentence $\theta$ s.t. $A+\theta$ is a $\breve{\Gamma}$-conservative extension of $A \cup X$.

Proof. By Craig's theorem, we may assume that $X$ is primitive recursive. Let $\xi(x)$ be a PR binumeration of $X$. Then

(1) $P \cup X \uparrow m \vdash \forall z \leqslant \bar{m}(\xi(z) \rightarrow \Gamma-\operatorname{true}(z))$.

By Lemma 2 , there is a $\Gamma$ sentence $\theta$ s.t.

(2) $A+\theta \vdash \xi(\bar{\varphi}) \rightarrow \Gamma$-true $(\bar{\phi})$,

(3) $A+\theta \dashv \check{\Gamma}^{A} \cup \cup\{\xi(\bar{q}) \rightarrow \Gamma$-true $(\bar{q}): q \in \omega\}$.

From (2) it follows that $A+\theta \vdash X$. Suppose $\psi$ is $\check{\Gamma}$ and $A+\theta \vdash \psi$. Then, by (1) and (3), $A \cup X \vdash \psi$. Thus $A+\theta \dashv \check{\Gamma} A \cup X$.

A closely related result is the following

THEOREM 5. Let $X$ be an r.e. set of $\Gamma$ sentences and let $Y$ be any r.e. set s.t. $A \cup X \forall \psi$ for every $\psi \in Y$. Then there is a $\Gamma$ sentence $\theta$ s.t. $A \cup X \dashv A+\theta \forall \psi$ for every $\psi \in Y$.

Proof. We may assume that $X$ and $Y$ are primitive recursive. Let $\xi(x)$ and $\eta(x)$ be PR binumerations of $X$ and $Y$, respectively.

Case 1. $\Gamma=\Pi_{n+1}^{0}$. Let $\theta$ be s.t.

$P \vdash \theta \leftrightarrow \forall y\left(\xi(y) \wedge \forall z u \leqslant y\left(\eta(z) \rightarrow \neg \operatorname{Prf}_{\alpha(x) \vee x=\bar{\theta}}(z, u)\right) \rightarrow \Pi_{n+1}^{0}-\operatorname{true}(y)\right)$. 
Suppose $\psi \in Y$ and $A+\theta \vdash \psi$. Then $P \cup X \vdash \theta$, whence $A \cup X \vdash \psi$, contrary to hypothesis. Thus $A+\theta \nvdash \psi$ for $\psi \in Y$. But then it follows that $A+\theta \vdash X$.

Case 2. $\Gamma=\Sigma_{n+1}^{0}$. Let $\theta$ be s.t.

$$
\begin{gathered}
P \vdash \theta \leftrightarrow \exists y\left(\exists z u \leqslant y\left(\eta(z) \wedge \operatorname{Prf}_{\alpha(x) \vee x=\bar{\theta}}(z, u)\right) \wedge\right. \\
\left.\forall z \leqslant y\left(\xi(z) \rightarrow \Sigma_{n+1}^{0} \text { true }(z)\right)\right) .
\end{gathered}
$$

The verification that $\theta$ is as claimed is now straightforward.

Guaspari [2] observed that $\operatorname{Cons}(\Gamma, A)$ is $\Pi_{2}^{0}$ and that Theorem 1 implies that $\Gamma \cap \operatorname{Cons}(\check{\Gamma}, A)$ is not r.e. and suggested the problem of classifying these sets. A complete solution (and more) follows from our next theorem. Partial results have been obtained by Hájek [4], Quinsey [13] and Solovay [16] using more complicated methods (cf. also [14]). Let

$$
\operatorname{Cons}(\Gamma, Y, A)=\{\varphi \text { : for every } \psi \in \Gamma \text {, if } A+\varphi \vdash \psi \text {, then } \psi \in Y\} \text {. }
$$

THEOREM 6. Suppose $\Gamma \neq \Pi_{1}^{0}$ and $Y$ is r.e. and mono-consistent with $P$. Then to any $\Pi_{2}^{0}$ set $X$, there is a $\Gamma$ formula $\xi(x)$ s.t.

(i) if $k \in X$, then $\xi(\bar{k}) \in \operatorname{HCons}(\breve{\Gamma}, A)$,

(ii) if $k_{r} \notin X, r \leqslant p$, then $\vee_{r \leqslant p} \xi\left(\bar{k}_{r}\right) \notin \operatorname{Cons}\left(\Pi_{1}^{0}, Y, A\right) \cup \operatorname{Cons}\left(\Sigma_{1}^{0}, Y, A\right)$.

To prove this we need the following

LEMMA 5. Suppose $X$ and $Y$ are r.e. and $Y$ is mono-consistent with $P$. Then there is a $\Sigma_{1}^{0}$ formula $\xi_{0}(x)$ and $a \Pi_{1}^{0}$ formula $\xi_{1}(x)$ s.t.

(i) $P \vdash \xi_{0}(\bar{k}) \rightarrow \xi_{1}(\bar{k})$,

(ii) if $k \in X$, then $P \vdash \xi_{0}(\bar{k})$,

(iii) if $k_{r} \notin X, r \leqslant p$, then $\vee_{r \leqslant p} \xi_{1}\left(\bar{k}_{r}\right) \notin Y$.

Proof. Let $\rho(x, y)$ and $\sigma(x, y)$ be PR binumerations of relations $R(k, m)$ and $S(k, m)$ s.t. $X=\{k: \exists m R(k, m)\}$ and $Y=\{k: \exists m S(k, m)\}$ and let $\xi_{1}(x)$ be s.t.

$$
P \vdash \xi_{1}(\bar{k}) \leftrightarrow \forall u(\chi(u) \rightarrow \exists z \leqslant u \rho(\bar{k}, z)),
$$

where $\chi(u)$ is the formula

$$
\exists v \leqslant u\left(\sigma(v, u) \wedge \text { “ } v \text { is of the form } \underset{r \leqslant p}{\vee} \xi_{1}\left(\bar{k}_{r}\right) " \wedge \forall w \leqslant u \forall q \leqslant p \neg \rho\left(k_{q}, w\right)\right) .
$$

Finally let $\xi_{0}(x)$ be s.t.

$$
P \vdash \xi_{0}(\bar{k}) \leftrightarrow \exists z(\rho(\bar{k}, z) \wedge \forall u \leqslant z \neg \chi(u)) .
$$

Then (i) is immediate. Next we show that for every $m, \chi(\bar{m})$ is false. Suppose $\chi(\bar{m})$ is true. Then

$$
P \vdash \xi_{1}(\bar{k}) \rightarrow \exists z \leqslant \bar{m} \rho(\bar{k}, z) .
$$

Moreover there are $k_{r}, r \leqslant p$, s.t. $\vee_{r \leqslant p} \xi_{1}\left(\bar{k}_{r}\right) \in Y$ and $P \vdash \neg \rho\left(\bar{k}_{r}, \bar{s}\right)$ for $r \leqslant p$ and $s \leqslant m$. But then $P \vdash \neg \vee_{r \leqslant p} \xi_{1}\left(\bar{k}_{r}\right)$, a contradiction. Thus $\chi(\bar{m})$ is false. But then (ii) and (iii) follow at once, the latter, since we may assume that $S(m, n)$ only if $m \leqslant n$. 
Proof of Theorem 6. We may assume that if $\varphi \in Y$ and $P \vdash \varphi \rightarrow \psi$, then $\psi \in Y$. Let $R(k, m)$ be an r.e. relation s.t. $X=\{k: \forall m R(k, m)\}$. By Lemma 5 , there is a $\Sigma_{1}^{0}$ formula $\rho_{0}(x, y)$ and a $\Pi_{1}^{0}$ formula $\rho_{1}(x, y)$ s.t.

(1) $P \vdash \rho_{0}(\bar{k}, \bar{m}) \rightarrow \rho_{1}(\bar{k}, \bar{m})$,

(2) if $R(k, m)$, then $P \vdash \rho_{0}(\bar{k}, \bar{m})$,

(3) if not $R(k, m), m \leqslant p$, then $\vee_{m \leqslant p} \rho_{1}(\bar{k}, \bar{m}) \notin Y$.

By Lemma 2 , there is a $\Gamma$ formula $\xi(x)$ s.t.

(4) $A+\xi(\bar{k}) \vdash \rho_{0}(\bar{k}, \bar{m})$,

(5) if $B \dashv A$, then $B+\xi(\bar{k}) \dashv \check{\Gamma} B \cup\left\{\rho_{0}(\bar{k}, \bar{q}): q \in \omega\right\}$.

Now (i) follows from (2) and (5) and (ii) follows from (1), (3) and (4).

Corollary 2. The sets $\Gamma \cap \operatorname{Cons}(\breve{\Gamma}, A)$, where $\Gamma \neq \Pi_{1}^{0}, \Pi_{2}^{0} \cap \operatorname{Cons}\left(\Sigma_{1}^{0}, A\right)$, and $\Sigma_{2}^{0} \cap \operatorname{Cons}\left(\Sigma_{1}^{0}, A\right)$ are complete $\Pi_{2}^{0}$ sets.

If $A$ is $\Sigma_{1}^{0}$-sound, then $\Pi_{1}^{0} \cap \operatorname{Cons}\left(\Sigma_{1}^{0}, A\right)$ is $\Pi_{1}^{0}$. However, Quinsey [13] has shown that if $A$ is not $\Sigma_{1}^{0}$-sound, then this set is a complete $\Pi_{2}^{0}$ set.

There are results similar to Theorem 6 and Corollary 2 for the sets $\Gamma \cap$ $\operatorname{DCons}(\breve{\Gamma}, A)$ but at this point they are quite easy and are therefore omitted.

The following corollary is applied in [10].

CoRollary 3. If $\Gamma \neq \Pi_{1}^{0}$, then $Z=\{\varphi: \exists \psi \in \Gamma \cap \operatorname{Cons}(\breve{\Gamma}, A)(A+\psi \vdash \varphi)\}$ is a complete $\Sigma_{3}^{0}$ set.

Proof. Clearly $Z$ is $\Sigma_{3}^{0}$. Let $X$ be any $\Sigma_{3}^{0}$ set and let $R(k, m)$ be a $\Pi_{2}^{0}$ relation s.t. $X=\{k: \exists m R(k, m)\}$. By Theorem 6, there is a $\Gamma$ formula $\rho(x, y)$ s.t.

if $R(k, m)$, then $\rho(\bar{k}, \bar{m}) \in \operatorname{Cons}(\breve{\Gamma}, A)$,

if not $R(k, m), m \leqslant p$, then $\vee_{m \leqslant p} \rho(\bar{k}, \bar{m}) \notin \operatorname{Cons}(\check{\Gamma}, A)$.

Let $Y_{k}=\{\neg \rho(\bar{k}, \bar{m}): m \in \omega\}$. Then, by (the proof of) Theorem 4, there is a formula $\eta(x)$ s.t. $A \cup Y_{k} \dashv A+\eta(\bar{k}) \dashv \breve{r} A \cup Y_{k}$. It follows that $X=\{k: \neg \eta(\bar{k}) \in Z\}$ and so the proof is complete.

Suppose $X$ is r.e. and let $Y=\{\varphi: A+\varphi \vdash X\}$. Clearly $Y$ is r.e. unless $X$ is infinite over $A$ in the sense that $A \cup X \uparrow k \sharp X$ for every $k$.

Theorem 7. Suppose $X$ is r.e., bounded, and infinite over A. Then $Y=\{\varphi$ : $A+\varphi \vdash X\}$ is a complete $\Pi_{2}^{0}$ set.

This was proved independently by Christian Bennet. In fact, the proof below is due to him and is presented here with his permission.

Proof. As usual we may assume that $X$ is primitive recursive. Let $\xi(x)$ be a PR binumeration of $X$. Let $n$ be s.t. $X \subseteq \Pi_{n+1}^{0}$. Next let $Z$ be any $\Pi_{2}^{0}$ set and let $R(k, m)$ be an r.e. relation s.t. $Z=\{k: \forall m R(k, m)\}$. By Theorem 1, there is a formula $\rho(x, y)$ s.t.

(1) if $R(k, m)$, then $A \vdash \rho(\bar{k}, \bar{m})$,

(2) if not $R(k, m)$, then $\neg \rho(\bar{k}, \bar{m}) \in \operatorname{Cons}\left(\Sigma_{n+2}^{0}, A\right)$.

Let $\eta(x)$ be the formula

$$
\forall z\left(\xi(z) \wedge \forall u \leqslant z \rho(x, u) \rightarrow \Pi_{n+1}^{0}-\operatorname{true}(z)\right) .
$$

It suffices to show that $Z=\{k: \eta(\bar{k}) \in Y\}$. If $k \in Z$, then, by (1), $A+\eta(\bar{k}) \vdash \xi(\bar{\varphi})$ $\rightarrow \Pi_{n+1}^{0}$-true $(\bar{\varphi})$ for every $\varphi$, whence $\eta(\bar{k}) \in Y$. Next suppose $k \notin Z$. Let $m$ be s.t. 
not $R(k, m)$. Then $A \cup X \uparrow m+\neg \rho(\bar{k}, \bar{m}) \vdash \eta(\bar{k})$. Let $\varphi \in X$. If $A+\eta(\bar{k}) \vdash \varphi$, then $A+\neg \rho(\bar{k}, \bar{m}) \vdash-X \uparrow m \rightarrow \varphi$, whence, by (2), $A \cup X \uparrow m \vdash \varphi$. But, $X$ being infinite over $A$, this fails for some $\varphi \in X$. It follows that $\eta(\bar{k}) \notin Y$.

2. Applications to interpretability. We write $S \leqslant T$ to signify that $S$ is interpretable in $T$. Most applications of partially conservative sentences to interpretability are based on the following (cf. $[2,3,9]$ )

LEMma 6. If $S, T$ are r.e. reflexive extensions of $P$, then $S \leqslant T$ iff $S \dashv_{\Pi_{1}^{0}} T$.

For brevity we assume from now on that $A$ and $B$ are essentially reflexive.

From Corollary 1 and Lemma 6 we get at once the following result essentially due to Hájek [3] (cf. also [5, 9, 14, 15, 16]).

THEOREM 8. If $X$ is r.e. and mono-consistent with $A$, then there is a $\Sigma_{1}^{0}$ sentence $\varphi$ s.t. $A+\varphi \leqslant A$ and $\varphi \notin X$.

Similarly, Theorem 2 yields the following (cf. [9])

THEOREM 9. If $X$ is r.e. and mono-consistent with $A$, then there is a sentence $\theta$ s.t. $A+\theta^{i} \leqslant A$ and $\theta^{i} \notin X, i=0,1$.

Theorem 8 was originally proved to give an example of a sentence $\varphi$ s.t. $\mathrm{ZF}+\varphi \leqslant \mathrm{ZF}$ and GB $+\varphi \nless \mathrm{GB}$ and this follows at once, since $\{\varphi$ : GB $+\varphi \leqslant \mathrm{GB}\}$ is r.e., GB being finite and mono-consistent with ZF. Similarly, Theorem 9 yields a sentence $\theta$ s.t. $\mathrm{ZF}+\theta^{i} \leqslant \mathrm{ZF}$ and $\mathrm{GB}+\theta^{i} \nless \mathrm{GB}, i=0,1$.

Our next result, which is an immediate consequence of Theorem 3 , answers a question raised by Orey [12].

THEOREM 10. There are sentences $\varphi_{i}$ s.t. $A+\varphi_{i} \leqslant A, A+\varphi_{0} \wedge \varphi_{1} \nless A, A+$ $\neg \varphi_{i} \nLeftarrow A$, and $A+\neg \varphi_{0} \vee \neg \varphi_{1} \leqslant A, i=0,1$. [9])

Let $A \equiv B$ mean that $A \leqslant B \leqslant A$. Then from Theorem 4 we get ((i) is proved in

THEOREM 11. (i) If $A \dashv B$, then there is a sentence $\theta$ s.t. $A+\theta \equiv B$.

(ii) If $X$ is an r.e. set of $\Sigma_{1}^{0}$ sentences, then there is a $\Sigma_{1}^{0}$ sentence $\sigma$ s.t. $A+\sigma \equiv A \cup X$.

The following corollary answers a question suggested by Švejdar [17]. $\psi$.

COROllary 4. There is a $\Sigma_{1}^{0}$ sentence $\sigma$ s.t. $A+\sigma \not \equiv A+\psi$ for every $\Pi_{1}^{0}$ sentence

Proof. There is an r.e. set $X$ of $\Sigma_{1}^{0}$ sentences s.t. $A \cup X \nless A \cup X \uparrow m$ for every $m$ (cf. [9]). By Theorem 11(ii), there is a $\Sigma_{1}^{0}$ sentence $\sigma$ s.t. $A+\sigma \equiv A \cup X$. Let $\psi$ be any $\Pi_{1}^{0}$ sentence and suppose $A+\sigma \equiv A+\psi$. Then, by Lemma 6 , there is an $m$ s.t. $A \cup X \uparrow m \vdash \psi$. But then $A \cup X \leqslant A+\sigma \leqslant A+\psi \dashv A \cup X \uparrow m$, whence $A \cup X \leqslant$ $A \cup X \uparrow m$, a contradiction.

The following result which was first proved by Solovay [16] (cf. also [2, 9, 14]) answers a question of Hájek [3]. It is an immediate consequence of Lemma 6 and Theorem 6 with $Y=\operatorname{Th}(B)$. 
THEOREM 12. If $A \leqslant B$, then $\Sigma_{1}^{0} \cap\{\varphi: A+\varphi \leqslant B\}$ is a complete $\Pi_{2}^{0}$ set.

If there is a $\Pi_{1}^{0}$ sentence $\theta$ s.t. $B \vdash \theta$ and $B \leqslant A+\theta$, then, by Lemma $6,\{\varphi$ : $B \leqslant A+\varphi$ \} is r.e. In contrast to this we have (cf. [9])

THEOREM 13. If there is no $\Pi_{1}^{0}$ sentence $\theta$ s.t. $B \vdash \theta$ and $B \leqslant A+\theta$, then $\{\varphi$ : $B \leqslant A+\varphi\}$ is a complete $\Pi_{2}^{0}$ set.

Proof. By assumption, the set of $\Pi_{1}^{0}$ sentences provable in $B$ is infinite over $A$. Moreover $B \leqslant A+\varphi$ iff $A+\varphi \vdash X$. Now apply Theorem 7 .

Suppose e.g. $\sigma$ is as in Corollary 4. Then, by Theorem 13, $\{\varphi: A+\sigma \leqslant A+\varphi\}$ is a complete $\Pi_{2}^{0}$ set.

\section{REFERENCES}

1. S. Feferman, Arithmetization of metamathematics in a general setting, Fund. Math. 49 (1960), 33-92.

2. D. Guaspari, Partially conservative extensions of arithmetic, Trans. Amer. Math. Soc. 254 (1979), 47-68.

3. P. Hajek, On interpretability in set theories, Comment. Math. Univ. Carolin. 12 (1971), 73-79; II, 13 (1972), 445-455.

4. I On partially conservative extensions of arithmetic, Logic Colloquium '78, North-Holland, Amsterdam, 1979, pp. 225-234.

5. M. Hájková and P. Hajek, On interpretability in theories containing arithmetic, Fund. Math. 76 (1972), 131-137.

6. D. Jensen and A. Ehrenfeucht, Some problems in elementary arithmetics, Fund. Math. 92 (1976), 223-245.

7. G. Kreisel, On weak completeness of intuitionistic predicate logic, J. Symbolic Logic 27 (1962), 139-158.

8. G. Kreisel and A. Lévy, Reflection principles and their use for establishing the complexity of axiomatic systems, Z. Math. Logik Grundlag. Math. 14 (1968), 97-142.

9. P. Lindström, Some results on interpretability, Proc. 5th Scandinavian Logic Sympos. (Aalborg, 1979), 1979, pp. 329-361.

10. On certain lattices of degrees of interpretability, Notre Dame J. Formal Logic (to appear).

11. On faithful interpretability (to appear).

12. S. Orey, Relative interpretations, Z. Math. Logik Grundlag. Math. 7 (1961), 146-153.

13. J. Quinsey, Sets of $\Sigma_{k}$-conservative sentences are $\Pi_{2}^{0}$ complete, J. Symbolic Logic 46 (1981), 442 (abstract).

14. C. Smoryński, Calculating self-referential statements-Guaspari sentences of the first kind, J. Symbolic Logic 46 (1981), 329-344.

15. __ Fifty years of self-reference, Notre Dame J. Formal Logic 22 (1981), 357-374.

16. R. Solovay, On interpretability in set theories (to appear).

17. V. Svejdar, Degrees of interpretability, Comment. Math. Univ. Carolin. 19 (1978), 789-813.

Department of Philosophy, University of Göteborg, Göteborg, SWeden 